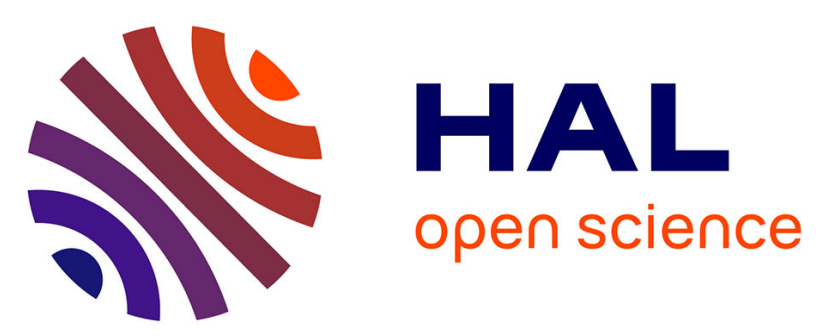

\title{
Molecular and crystal structure of 7-fold V-amylose complexed with 2-propanol
}

Y. Nishiyama, K. Mazeau, M. Morin, M.B. Cardoso, H. Chanzy, J.L. Putaux

\section{To cite this version:}

Y. Nishiyama, K. Mazeau, M. Morin, M.B. Cardoso, H. Chanzy, et al.. Molecular and crystal structure of 7-fold V-amylose complexed with 2-propanol. Macromolecules, 2010, 43 (20), pp.8628-8636. $10.1021 / \mathrm{ma101794w}$. hal-00546478

\section{HAL Id: hal-00546478 https://hal.science/hal-00546478}

Submitted on 1 Dec 2021

HAL is a multi-disciplinary open access archive for the deposit and dissemination of scientific research documents, whether they are published or not. The documents may come from teaching and research institutions in France or abroad, or from public or private research centers.
L'archive ouverte pluridisciplinaire HAL, est destinée au dépôt et à la diffusion de documents scientifiques de niveau recherche, publiés ou non, émanant des établissements d'enseignement et de recherche français ou étrangers, des laboratoires publics ou privés. 


\title{
Molecular and crystal structure of 7-fold V-amylose complexed with 2-propanol
}

\author{
Yoshiharu Nishiyama, Karim Mazeau, Morgane Morin, Mateus B. Cardoso, \\ Henri Chanzy and Jean-Luc Putaux*
}

Centre de Recherches sur les Macromolécules Végétales (CERMAV-CNRS), BP 53, F-38041 Grenoble cedex 9, France - affiliated with the Université Joseph Fourier and member of the Institut de Chimie Moléculaire de Grenoble

* Corresponding author: jean-luc.putaux@cermav.cnrs.fr

Published in: Macromolecules 43 (2010), 8628-8636

DOI: $10.1021 / \mathrm{ma101794w}$ 


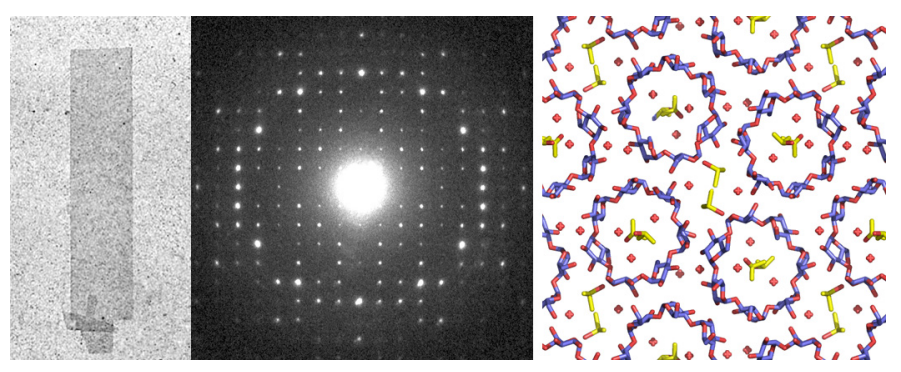

\begin{abstract}
Single crystals of amylose $\mathrm{V}_{2 \text {-propanol, }}$ i.e., amylose co-crystallized with water and 2-propanol, were prepared from synthetic linear amylose. When observed under frozen hydrated conditions, these crystals yielded base-plane electron diffraction diagrams containing more than 100 independent diffraction spots, whose intensities were used to solve the crystal structure of this complex. The molecular and crystal structure of amylose $\mathrm{V}_{2 \text {-propanol }}$ clearly indicated that the amylose molecules were organized in 7-fold left-handed helices, with 2-propanol and water molecules located as guests both within and between the helices. The $\mathrm{V}_{\text {2-propanol }}$ unit cell contains 4 helices, distributed in two antiparallel pairs of parallel helices organized along the $P 2{ }_{1} 2_{1} 2_{1}$ symmetry. The helices are organized along alternating motifs of 4 helices in a close-packed hexagonal arrangement together with 4 others in a nearly square organization surrounding a central column of water and 2-propanol. Whereas the location of the amylose helices is well established in the unit cell, the coordinates of the guest molecules could not be defined with certainty, most likely due to a positional disorder. A tentative model of the guest molecule distribution is presented, which consists of 2 2-propanol and 2 water molecules within the helical cavity together with 4 water molecules and 2 2-propanol molecules between the helices. The mobility of the guest and its description as a continuum, rather than at fixed crystallographic positions, explain why so many structures isomorphous to $\mathrm{V}_{\text {2-propanol }}$ can be obtained with different guest molecules, while yielding similar electron diffraction diagrams.
\end{abstract}

Keywords: Helical amylose; single crystal, inclusion complex, 2-propanol, electron diffraction, structure analysis 


\section{Introduction}

Amylose, the linear $\alpha(1 \rightarrow 4)$-D-glucan component of starch, forms complexes with a long list of inorganic and organic guests. ${ }^{1-4}$ This remarkable property in the world of polysaccharides explains why so many allomorphs are found for crystalline amylose. In semi-crystalline starch granules, the short linear $\alpha(1 \rightarrow 4)$-D branches of amylopectin intertwine into double helices and associate to form crystallites together with water molecules. ${ }^{5}$ Amylose is generally amorphous in the granules but, to some extent, may also participate to crystallites. ${ }^{6}$ In the starch granules, one identifies two main allomorphs, namely A and B, chiefly found in cereal and tuber starches, respectively. ${ }^{5}$ The C-type, found in bean or root starches, has been shown to be a mixture of $\mathrm{A}$ and B allomorphs. ${ }^{5} \mathrm{~A}-$ and B-amyloses are also obtained by recrystallization from either pure aqueous amylose solutions (for B) or when a small amount of organic precipitant such as ethanol is added to the solutions (for A). ${ }^{7-9}$ When larger amounts of precipitant are added to solutions of amylose in hot water, amylose invariably crystallizes as single helices but there are many ways in which these helices can pack together, depending on the nature of the precipitant. ${ }^{5}$ These multiple possibilities explain why so many single helical amylose allomorphs can be found. These crystals, categorized under the generic name of $\mathrm{V}$-amylose, ${ }^{10}$ differ from one another by their unit cell parameters and symmetry elements, as well as by the inclusion of guests within the amylose helical cavity and/or between the helices. ${ }^{5}$ The $\mathrm{V}$ allomorph is rarely encountered in nature except when amylose forms complexes with lipids in amylose-rich starch granules. ${ }^{11}$

Some crystal structures of V-amylose have been resolved using the data collected by either X-ray diffraction on fibers or electron diffraction on single crystals. Among these structures, the hexagonal hydrated $\mathrm{V}$-amylose - or $\mathrm{V}_{\mathrm{H}}$ amylose ${ }^{12-14}$ - as well as the blue complex of amylose with iodine - or $\mathrm{V}_{\text {iodine }}{ }^{15}$ - have been defined as tight hexagonal packings of 6-fold left-handed helices, presenting a repeat distance of $0.8 \mathrm{~nm}$ along the helix axis. Water molecules, in the case

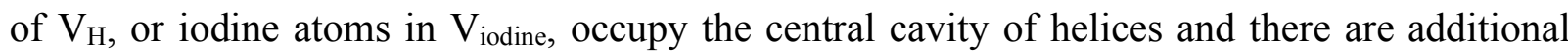
water molecules in the inter-helical spaces. The 6-fold left-handed helical system has also been proposed for the pseudo-tetragonal $\mathrm{V}_{\text {DMSO }}$ crystals. In these, however, the packing of the amylose helices is no longer tight since, in addition to the intra-helical DMSO, there are also some DMSO molecules located between the helices, which results in fairly large unit cell parameters in the plane perpendicular to the fiber/helical axis. ${ }^{16}$ For several other V-amylose complexes, diffraction diagrams have been recorded, ${ }^{5,17}$ but no three-dimensional model is available yet.

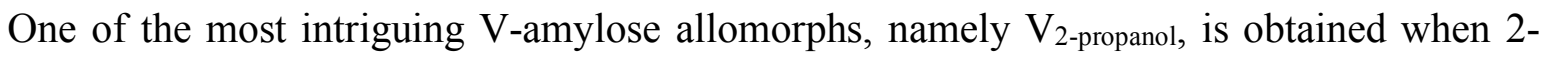
propanol is added to hot dilute aqueous solutions of amylose. Under optimum conditions, the 
resulting precipitate consists of thin rectangular micron-sized single-crystals ${ }^{17-20}$ that yield rich electron diffraction diagrams provided that their recording is achieved under cryo-electron microscopy conditions. ${ }^{19,20}$ Quite remarkably, single crystals that yield similar electron diffraction diagrams as the one of $\mathrm{V}_{2 \text {-propanol }}$ are also obtained with many precipitants such as acetone, thymol, $t$-butanol as well as a variety of food flavoring agents, to name just a few. ${ }^{5,20-22}$ The crystalline complexes of amylose with flavor guests present a great interest since these molecules, which are incorporated within the crystalline lattice, may be released during food consumption. ${ }^{23}$ It is not clear at present whether these guests are located within or between the amylose helices. The location of the guests should have a strong impact on their release ability, since their binding capacity should be different whether they occupy or not the helical cavity.

Hypotheses for the three-dimensional structure of the crystals of the $\mathrm{V}_{\text {2-propanol family have }}$ been proposed. From diffraction data, it was found that the helical repeat was of around $0.8 \mathrm{~nm}$, i.e., the same as that of the helical 6-fold $\mathrm{V}_{\mathrm{H}}$ or $\mathrm{V}_{\text {iodine }}$ complexes. ${ }^{12-15}$ Despite this similarity, the other unit cell parameters deduced from X-ray diffraction data could be fitted with helices having an external diameter of $1.5 \mathrm{~nm}$, corresponding to 7-fold helices, ${ }^{18,24-26}$ as opposed to $1.3 \mathrm{~nm}$ for the 6-fold helix of $\mathrm{V}_{\mathrm{H}}$ amylose. However, the occurrence of 7-fold helices was questioned since the crystals of $\mathrm{V}_{2 \text {-propanol }}$ could be readily converted in the solid-state to the well-established 6fold $\mathrm{V}_{\mathrm{H}}$ structure by either annealing in 2-propanol or by washing in methanol. ${ }^{18,19}$ Thus, in absence of a conclusive three-dimensional structure, the diffraction data of $\mathrm{V}_{2 \text {-propanol }}$ crystals could also be accounted for by a system of 6-fold amylose helices separated by a layer of guest molecules and therefore different from the close-packed arrangement of 7-fold helices. ${ }^{19,21}$

The present work was therefore undertaken to resolve this dilemma, by attempting a determination of the molecular and crystal structure of the $\mathrm{V}_{2 \text {-propanol }}$ crystal. For this, batches of $\mathrm{V}_{2 \text {-propanol }}$ single crystals were prepared and their electron diffraction diagrams used for a structure refinement based on conformational and packing energy minimization analyses, combined with classical crystalline polymer structure refinement.

\section{Experimental}

Crystal preparation. Synthetic amylose with an average degree of polymerization $(D P)$ of 100, a gift from Dr. Gessler (Free University of Berlin, Germany), was dispersed in water at a concentration of $0.5 \mathrm{~g} / \mathrm{L}$. The solutions were submitted to nitrogen bubbling for $20 \mathrm{~min}$, sealed in a glass vial, heated for $30 \mathrm{~min}$ at $150^{\circ} \mathrm{C}$ by immersion in an oil bath, cooled to $90^{\circ} \mathrm{C}$ and filtered through a $0.2 \mu \mathrm{m}$ pre-heated filter. A pre-heated solution of 2-propanol was added to reach a waterto-2-propanol ratio of $65 / 35(\mathrm{v} / \mathrm{v})$. After gentle shaking, the mixtures were allowed to slowly cool 
in a Dewar bottle. Crystallization occurred overnight. The crystals were rinsed by sedimentation or very slow centrifugation in a 50/50 (v/v) mixture of water and 2-propanol.

Transmission electron microscopy (TEM). Drops of dilute crystal suspensions were deposited onto glow-discharge carbon-coated copper grids and after $1 \mathrm{~min}$, the liquid in excess was blotted with filter paper. The grids were then mounted on a Gatan 626 cryoholder, quenchfrozen in liquid nitrogen, transferred into the microscope and cooled to $-180^{\circ} \mathrm{C}$. The specimens were observed with a Philips CM200 'Cryo' microscope operating at $80 \mathrm{kV}$ for imaging and 200 $\mathrm{kV}$ for diffraction. Base-plane diffraction diagrams containing $h k 0$ reflections were recorded on $1 \mu \mathrm{m}^{2}$ circular areas of single crystals and the $d$-spacings were calibrated using a gold standard. Images were recorded on Kodak SO163 films and diffraction patterns on Fujifilm imaging plates, read with a Fujifilm BAS-1800II Bio-imaging Analyzer.

The intensity of diffraction spots was measured semi-automatically using a tailor-made program. The position of reflections 600 and 060 was first determined to calculate the 100 and 010 base vectors of the diagram. Square boxes were extracted around the diffraction spots detected at each point of the lattice defined by these base vectors. The size of the box $(16 \times 16$ pixels $^{2}$ ) was about three times the size of the spot. The intensity of each reflection was calculated by fitting the data with a Gaussian peak function and a linear background. In the case of saturated peaks, the Gaussian fit was done on the not-saturated base of the reflection. In view of the symmetry of the patterns, the intensities of $h k 0,-h k 0, h-k 0$ and $-h-k 0$ reflections were averaged. The diffraction patterns were normalized using the strongest intensities of each pattern as reference. A set of diffracted intensities was finally obtained by averaging the intensities over several diffraction patterns.

Molecular Modeling and Crystal Structure Determination. Strategy. Since electron diffraction diagrams similar to those obtained with the $\mathrm{V}_{\text {2-propanol }}$ crystal can be recorded when amylose is crystallized with many guest molecules having molecular geometries far different from that of 2-propanol, it seems reasonable to assume that the guests and their accompanying water molecules do not occupy periodic crystallographic positions. Rather, they are expected to fill up the voids located inside the amylose helices and in between in a statistical manner that can be approximated by a constant electron density (or constant electric potential for electron diffraction). Since the degree of freedom for the guest molecules is much higher than that of the rigid amylose helices, only these and not the guest molecules were considered to occupy periodic crystallographic positions within the unit cell at the start of the refinement. 
Building the Amylose Helices. Eighteen regular right- and left-handed amylose helices, having 6, 7 or 8 glucosyl residues per turn and the hydroxymethyl group either in gauche-gauche $(g g)$, gauche-trans $(g t)$ or trans-gauche $(t g)$ conformation were generated as follows. A master $\alpha$-D-glucosyl residue in the ${ }^{4} \mathrm{C}_{1}$ chair conformation, was built and its energy minimized using the Tripos 5.2 force field. ${ }^{27}$ This residue was used to propagate 6 regular helices with symmetries $P 6_{1}, P 6_{5}, P 7_{1}, P 7_{6}, P 8_{1}$ and $P 8_{7}$ using the classical parameters $n$ (number of residues per turn) and $h$ (rise per residue). ${ }^{28} P 6_{1}, P 7_{1}$, and $P 8_{1}$ correspond to right-handed 6-, 7-, and 8-fold helices whereas $P 6_{5}, P 7_{6}$ and $P 8_{7}$ are their left-handed counterparts. These helices will be referred to as $R 6, R 7, R 8, L 6, L 7, L 8$, respectively, in the following. The master glucosyl unit was then rotated around its virtual bond $\mathrm{O} 1-\mathrm{O} 4^{29}$ by 1-degree steps and the energy of the resulting helix calculated at each step. For the lowest energy models, 3 turns were made and a constraint of continuity was set on both ends of each helix to ensure that infinitely long helices could be propagated. For each of these 6 helices, the hydroxymethyl group of the glucosyl residues was positioned in the $\mathrm{tg}, \mathrm{gg}$ or $g t$ conformation and their energy minimized using the Universal Force Field ${ }^{30}$ of the Cerius package, ${ }^{31}$ leading to the 18 helices that were considered.

Exhaustive Search of Helix Positions in the Unit Cell. The packing of the 18 helices was investigated by positioning each of them into the unit cell, and generating their analogues using the $P 2{ }_{1} 2_{1} 2_{1}$ symmetry. Each helix had four degrees of freedom, three translational and one rotational around its helical axis. The center of the helix was stepped along the $a$ and $b$ axes by 0.01 fractions, corresponding to about $0.02 \mathrm{~nm}$, within $1 / 8^{\text {th }}$ of the $(a, b)$ projection of the unit cell. This was sufficient to cover all the possibilities, considering the symmetry of the unit cell. At each position, the helix was rotated around its helical axis by $5^{\circ}$ steps between 0 and $360^{\circ}$ and translated along the $c$-axis from 0 to $1.2 \AA$ with an interval of $0.3 \AA$. Only the non-bonded interaction was considered using Buckingham's potential ${ }^{32}$ with a cutoff of $1 \mathrm{~nm}$. At each translational position of the helical center, the lowest packing energy and corresponding rotation angle and translation along $c$ were saved. A contour map of these minima was then drawn.

Different methods for taking solvent scattering into account have been developed, especially for the resolution of protein structure from fiber diffraction data. ${ }^{33}$ It was shown that the implementation of these methods led to a substantial reduction of the calculated intensities of the diffracted peaks at low scattering angles. In principle, the Fourier transform of the excluded volume due to the atom in a continuous background is added to the conventional atomic scattering factor. Hard spheres or Gaussian shapes with van der Waals or empirical atomic radius have been proposed to describe the excluded volume of each atom. In the present study, we have chosen the Gaussian solution for the ease in calculation since crystallography software like 
SHELX $^{34}$ use Gaussian function series to approximate the atomic scattering factors. The calculation method and parameters of the effective atomic scattering factors used in this study are detailed in Supporting Information, Table S1.

The structure refinement, which was initially achieved at a $0.3 \mathrm{~nm}$ resolution, is based on the intensity of 71 independent reflections, which were averaged from the four equivalent quadrants on the base-plane electron diffraction pattern. Following the aforementioned protocol, a continuum of solvent density of $0.8 \mathrm{~g} / \mathrm{cm}^{3}$ - i.e., that of 2-propanol - was assumed. For each of these, electron diffraction diagrams were calculated and probed against the observed one. We have determined an $R_{2}$-factor, calculated as:

$$
R_{2}=1-\left(\frac{I_{\text {obs }} \boldsymbol{I}_{\text {calc }}}{\left\|\boldsymbol{I}_{\text {obs }}\right\| \times\left\|\boldsymbol{I}_{\text {calc }}\right\|}\right)^{2}
$$

where $\boldsymbol{I}_{\boldsymbol{o b s}}$ and $\boldsymbol{I}_{\text {calc }}$ correspond to the vectorial representation of the observed and calculated intensities. The components of the vector $\boldsymbol{I}$ are the intensities of the 71 reflections considered in the calculation: $\boldsymbol{I}=\left(I_{1}, I_{2}, I_{3}, \ldots, I_{i}, \ldots, I_{n}\right)$ with $\mathrm{n}=71$. The advantage of such mathematical description for the $R_{2}$-factor is that no regression procedure is required as opposed to the classical case, which needs a scaling factor to be refined. An $R_{2}$-factor map was then drawn within $1 / 8^{\text {th }}$ of the $(a, b)$ projection of the unit cell.

Structure refinement. From the superimposition of the packing energy maps and those of the $R_{2}$-factor maps, we identified the few potentially acceptable structures that were further processed by the SHELX-97 program, ${ }^{34}$ adapted for electron diffraction data, ${ }^{35}$ allowing the helices to relax. First, a unique scaling factor was used to match the observed intensities with the calculated ones. In a second step, this matching was improved by dividing the list of the diffraction intensities into subsets as function of their scattering angle and refining the scale factor for each of them, using the BASF scale factor protocol of SHELX.

Structure Refinement with Explicit Solvent Molecules. For the selected models, a Connolly algorithm ${ }^{36}$ was used to define the voids located either within the helical cavities or between the helices. 2-propanol and water molecules in arbitrary orientation were manually inserted into these voids and their position and orientation were refined by energy minimization, using the Cerius package. These solvated structures were then refined against the experimental diffraction dataset using SHELX. Reliability factors $R_{l}$ were calculated as:

$$
R_{l}=\frac{\sum|| F_{o b s}|-| F_{c a l c}||}{\sum\left|F_{o b s}\right|}
$$

where $F_{\text {obs }}$ and $F_{\text {calc }}$ are the observed and calculated structure factors, respectively. 


\section{Results}

The Crystals and their Base-Plane Diffraction Pattern. A group of typical $V_{2 \text {-propanol }}$ lamellar crystals is shown in Figure 1a. These crystals consist of a series of superimposed rectangular lamellae radiating from a common origin. As observed in earlier work, ${ }^{19}$ each lamella is about 3 to $4 \mu \mathrm{m}$-long, $1 \mu \mathrm{m}$-wide and about $10 \mathrm{~nm}$-thick. In areas of lamellar superposition, the crystals present a series of cracks that roughly run parallel to the long dimension of the crystals. These cracks, which are likely caused by the drying of the crystals in air or in the vacuum of the TEM, are absent when the crystals are observed under frozen-solvated conditions. Upon gentle shaking of the crystal suspension, individual lamellae such as the one shown in Figure 1b can be isolated and their electron diffraction pattern recorded. Figure 1c shows the center of a typical diagram recorded on a $1 \mu \mathrm{m}^{2}$ surface of the specimen. The original diagram (not shown) contains more than 400 diffraction spots, extending to a resolution of $0.2 \mathrm{~nm}$ and organized along a lattice defined by the two orthogonal axes $a^{*}$ and $b^{*}$. The diagram in Figure 1c, which is similar, but better resolved than those published earlier, ${ }^{5,18,20}$ presents spots of strong intensity on the $5^{\text {th }}$ and $6^{\text {th }}$ rows, whereas they are rather weak on rows 1 to 4 or beyond row $6 .{ }^{19}$ From the observation of symmetric diffraction spots, together with systematic absences of the odd reflections on the $a^{*}$ and $b^{*}$ axes, a projected structure having the two-dimensional space group $p_{g g}$ can be inferred, in agreement with earlier observations. ${ }^{19}$ After calibration, the rectangular cell parameters $a=2.826 \mathrm{~nm}$ and $b=2.950 \mathrm{~nm}$ were deduced, assuming, as shown earlier, ${ }^{19,21}$ that the $c$-axis was perpendicular to the $(a, b)$ base plane, with a repeat distance of $0.801 \mathrm{~nm}$. Taken together, these geometric factors and their symmetry indicate that the most reasonable three-dimensional space group for this structure is $P 2{ }_{1} 2{ }_{1}{ }_{1}$. A list of 108 independent intensities for reflections up to a $d$-spacing of $0.214 \mathrm{~nm}$, averaged from a series of patterns, is presented in Supporting Information Table S2.

Structure Determination and Refinement. Choosing the Amylose Helix. Examples of the right and left-handed 6-, 7- and 8-fold helices, taken from the 18 different amylose helices that were built for this work, are shown in Figure 2. Figures 3a-3c illustrate examples of the lowest energy packing of $L 6, L 7$ and $L 8$ rigid helices, projected on the unit-cell $(a, b)$ base plane and duplicated twice according to the $P 2_{1} 2_{1} 2_{1}$ space group, when the $2_{1}$ axes are located between the helices. Whereas the models in Figures 3a and 3b, for the 6-fold and 7-fold helices, respectively, seem acceptable without apparent collision, this is not the case for the 8-fold helices which show significant overlap. Table 1 summarizes important features of the packing models obtained with 
the 18 helices. In this table, the second column lists the minimum packing energy that can be calculated for the different helices, taken as rigid bodies, when they are rotated and translated within the unit cell. A study of this list shows that a structure based on 8-fold helices would present far too high packing energies, indicating that the four helices of the unit cell are colliding and therefore cannot fit into the cell volume. The minimum packing energy of the 6 models of 7-fold helices system appear more acceptable, even if the 7-fold models have better packing energy than their 6-fold counterpart. In the 7-fold family, the left-handed structures have lower packing energy than the right-handed ones and overall, the $L 7 g g$ stands out as being the most energetically favored. The third column in Table 1 lists the lowest $R_{2}$-factors resulting from the comparison between the observed and calculated intensity datasets, when the helices are translated and rotated within the unit cell and the guest is taken as a continuum of density of 0.8 $\mathrm{g} / \mathrm{cm}^{3}$ within the voids in the structures. This column clearly indicates that only the structures based on 7-fold helices yield acceptable calculated diffraction diagrams and thus, the 6-fold as well as the 8-fold helical structures can be ruled out altogether. In the list of 7-fold helices, the $L 7 g g$ and $L 7 g t$ stand out as presenting the lowest $R_{2}$-factors: 0.214 and 0.212 , respectively (calculated from 71 reflections with $d$-spacings higher than $0.3 \mathrm{~nm}$ ). However, the $R 7 g g$ and $R 7 g t$ can also be considered on the ground of their slightly higher packing energy $(-41.6$ and $-41.2 \mathrm{~kJ} / \mathrm{glucosyl}$ unit respectively) and their acceptable $R_{2}$-factor $(0.317$ and 0.307 , respectively). The selection of the helix center coordinates of the best 7-fold helices is exemplified in Figures 4a and $\mathbf{4 b}$ for the $\mathrm{L} 7 g g$ case. For this helix, Figure 4a presents the minima of the packing energy in a color map. In this figure, the lowest energy (arrowhead 1) of $-49.1 \mathrm{~kJ} /$ glucosyl unit corresponds to the helix centered at the fractional coordinates $\mathrm{x}=0.05$ and $\mathrm{y}=0.34$ defined along the unit cell parameters $a$ and $b$. In addition, there is a secondary energy minimum nearly as low (arrowhead 2) of $-48.8 \mathrm{~kJ} /$ glucosyl unit for a helix centered at $\mathrm{x}=0.05$ and $\mathrm{y}=0.17$. Figure $4 \mathrm{~b}$ presents the minima of the $R_{2}$-factor in a color map. In this map, the lowest $R 2$-factor (0.214) is observed for a helix centered at $\mathrm{x}=0.05$ and $\mathrm{y}=0.34$ (arrowhead). Taking the data in Figures $\mathbf{4 a}$ and $\mathbf{4 b}$ together, it appears that the coordinates of minima 1 in Figure 4a matches those of the minimum in Figure $\mathbf{4 b}$. Thus, these coordinates stand out as the only helix position for further crystallographic refinement. Maps (not shown), such as those shown in Figures $4 \mathbf{a}$ and $\mathbf{4 b}$ were also built for the $L 7 g t, R 7 t g$ an $R 7 g g$ structures. For each of these 4 structures, starting models for which both the packing energy and $R_{2}$-factor were minimal were selected for further refinement.

Structure Refinement. The four selected structures $\mathrm{L} 7 \mathrm{gg}, \mathrm{L7gt}, \mathrm{R} 7 \mathrm{gg}$ and $\mathrm{R} 7 \mathrm{tg}$ in their solvent continuum were subjected to a first SHELX refinement that allowed the helices to relax. The 
result of this refinement is shown in Table 2, which presents the $R_{l}$-factors of the four refined structures. Only the structure $L 7 g g$ appears to be amenable to further refinement as it presents an $R_{1}$-factor of 0.28 , whereas in the other three structures, the $R_{l}$-factor is above 0.4 . At this stage, a survey of the experimental and calculated electron diffraction diagrams of the $L 7 g g$ model (Figure 5) indicates that despite the fact that the two diagrams present a great deal of similarity, the reflections with $d$-spacings between 0.6 to $1.0 \mathrm{~nm}$ (zone 2 in Figure 5) have calculated intensities systematically larger than those of the corresponding ones in the observed diagrams. Conversely, there is a better match between the calculated and observed diagrams for reflection with $d$-spacings between 2.0 and $1.0 \mathrm{~nm}$ or those between 0.6 and $0.3 \mathrm{~nm}$ (zones 1 and 3, respectively, in Figure 5). This divergence likely results from the influence of the guest solvent that is not precisely taken into account in the calculated diffraction diagrams when using the continuum concept. To overcome this difficulty, we used the "BASF" scaling protocol of SHELX. In this procedure, the list of intensities was divided into 7 batches as a function of their scattering angle, and the scale factor was refined for each of them. A substantial improvement of the $R_{1}$-factor resulted: it decreased to 0.23 when using the 71 reflections $(0.303 \mathrm{~nm}$ resolution) and to 0.26 when the full dataset containing 108 reflections $(0.214 \mathrm{~nm}$ resolution) was considered (Table 2).

Figure 6a shows a projection perpendicular to the $c$-axis of the structure at this level of refinement. As opposed to the initial rigid helices (Figure 2b) that had a regular heptagonal projection on the $(a, b)$ plane, the refined relaxed helices have an oval shape. The volume susceptible to be occupied by the solvent can be defined by drawing Connolly surfaces at the contours of the relaxed helices (Figure 6b). One can tentatively fill these voids by positioning water and 2-propanol molecules and refining their position and orientation with the Cerius program, using packing energy considerations. An acceptable model after SHELX refinement is presented in Figure 6c. This model holds 2 2-propanol and 3 water molecules within the helical cavity together with 2 2-propanol and 4 water molecules between the helices. Remarkably, this structure presents $R_{1}$-factors of 0.18 and 0.10 calculated from 108 and 71 reflections respectively, and yields a crystal density of $1.42 \mathrm{~g} / \mathrm{cm}^{3}$, a figure that is consistent with the experimental density of these samples. ${ }^{19}$ Crystallographic parameters of this structure are listed in a CIF file given as Supporting Information and a selection of the geometric parameters is presented in Table 3. In this table, one can compare the conformational angles $\tau, \Phi, \Psi$ and the puckering azimuthal angle $\theta^{37}$ of the structure resulting from the final SHELX refinement with those of the preceding one, where the solvent was taken as continuum. In this comparison, one sees that despite a substantial improvement of the $R_{l}$-factor, the final structure appears to diverge from the one made of regular 
L7 helices. Whereas in the final structure, the glucosyl rings are not too deformed, there are substantial modifications in the hydroxymethyl group conformation: among the 7 residues, which initially were all in the $g g$ conformation, one of them (residue 4) has shifted toward a $g t$ conformation whereas two others (residues 6 and 7) are on the borderline between $g g$ and $t g$. Representations of the final structure, parallel and perpendicular to the amylose helix axes are shown in Figure 7. Figure 8 presents the observed electron diffraction diagram and the one calculated from the final model. In this figure, one sees that there is a close match between the two patterns and in particular the discrepancies that were outlined in Figure 5, are no longer present.

\section{Discussion}

One of the main results of the present study is to lift the ambiguity in the description of the

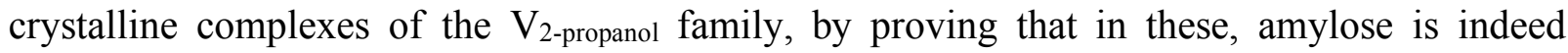
organized in the form of $L 7$ helices. Historically, a 7-fold structure had been suggested for the complex of amylose with tert-butanol, ${ }^{24,38}$ which yields crystals that are isomorphous to those of $\mathrm{V}_{2 \text {-propanol. }}{ }^{18}$ Such a proposal was also made by Takeo and Kuge who showed some similarities between the X-ray powder diagram of the dried tert-butanol amylose complex, and those of some $\beta$-cyclodextrin complexes. ${ }^{25}$ However, in their report, these authors suggested that the dried tertbutanol amylose complex was based on 7-fold amylose organized in a close-packed hexagonal system. In fact, such packing had been previously eliminated by Zaslow who clearly stated that it was not possible to have a hexagonal close packing of 7-fold helices. ${ }^{24}$ As an alternative to the 7-fold organization for the $\mathrm{V}_{2 \text {-propanol }}$ single crystals, we could show that these crystals had features in common with those based on 6-fold structures. ${ }^{19}$ In particular, the $\mathrm{V}_{2 \text {-propanol }}$ crystals could be readily converted into the $L 6 \mathrm{~V}_{\mathrm{H}}$ system without loosing their single crystal character. Such transition was obtained by either transferring the crystals into a methanol medium or heatannealing them in pure 2-propanol. Thus, as an alternative hypothesis to the 7-fold, the structure of $\mathrm{V}_{2 \text {-propanol }}$ crystals could be conceived as being built of $L 6$ helices separated by layers of guest solvent. In particular, the 2-propanol molecules could be located between the helices and not inside, since 2-propanol is too large to fit into the cavity of a 6-fold amylose helix. The present study rules out our earlier hypothesis and demonstrates that, at least in their wet state, the $V_{2-}$ propanol crystals are indeed made of $L 7$ helices with 2-propanol and water molecules organized inside the helical cavity as well as in pockets between the helices. In agreement with the comment of Zaslow ${ }^{24}$ and unlike the proposal of Takeo and Kuge, ${ }^{25}$ we see in Figures 6a to $6 \mathbf{c}$ that the 7-fold helices are not organized in close-packed hexagonal arrangement where each helix would 
be in contact with 6 neighbors. In fact, the packing of the helices consists of alternating motifs of 4 helices organized in a close-packed hexagonal arrangement together with 4 helices in a nearly square arrangement surrounding a central column of water and 2-propanol (Figure 9). It is thanks to these two motifs that the tiling of the present 7-fold helices can be regular.

A comparison of the $L 7 g g$ amylose helices before and after refinement is instructive as it shows that the heptagonal $L 7$ amylose helices, which were initially built symmetrically and already gave the best $R_{2}$-factor before refinement, became somewhat distorted during the SHELX refinement cycles. This distortion is well observed in the projection of the helices on the $(a, b)$ plane of the unit cell: the initial rigid helices had a projection that could be inscribed in a circle (Figure 3b), but after the relaxation induced by the refinement, the relaxed helices adopted an elliptical cross-section (Figure 6a). In fact, elliptical amylose helices had already been suggested by Simpson et al. ${ }^{26}$ for their crystalline complex of amylose with DMSO, on the ground that the intra-helical DMSO, which was not spatially cylindrical, would distort the helix cavity. Such reasoning may account for the ellipticity of the present amylose helices. Interestingly, the comparison between Figures 6a and $\mathbf{6 c}$ indicates that the projection of the helical amylose skeleton is roughly established in the initial SHELX refinement, when the guest solvent was still taken as a continuum (Figure 6a). The further steps of refinement did not substantially alter the structure of the skeleton, but had drastic effects on the conformation of the hydroxymethyl groups, one of them being refined to the $g t$ situation whereas for two others, the refinement led to conformations intermediate between $g g$ and $\operatorname{tg}$ (Table 3).

One of the main difficulties in solving the structure of the crystals of $\mathrm{V}_{2-\text { propanol }}$ has been to account for the 2-propanol and water molecules, which are filling the intra- and inter-helical cavities of the structure, without occupying crystallographic positions. It is known that such guest molecules have a significant influence on the intensity of the diffraction spots, ${ }^{33}$ but only empirical methods can be used to subtract the ligand contribution from the diffraction datasets. Here, we have successively used three methods to account for the guest molecules, namely its contribution as a continuum of density $0.8 \mathrm{~g} / \mathrm{cm}^{3}$, the use of adjustable scaling factors to correct the observed intensities and, finally, the insertion of explicit guest molecules in the intra- and inter-helical cavities of the structure. Obviously, the succession of these procedures has improved the match between the observed and calculated diffraction diagrams (see Table 2 and Figure 8). Nevertheless, the precise positioning of explicit water and 2-propanol molecules, as in Figures 6c and 8, must be considered as one local solution among many others differing from one another by the distribution of the guest molecules. In this respect, the ${ }^{13} \mathrm{C}$ solid-state NMR spectrum of crystalline $\mathrm{V}_{2 \text {-propanol }}$ is informative. ${ }^{39}$ Only the resonances attributed to the carbon 
atoms of crystalline amylose are present, but not those of 2-propanol, since they are relaxing too fast to be detected under the recording conditions. This behavior agrees with the solvent mobility deduced from our structural analysis. Remarkably, the ${ }^{13} \mathrm{C}$ solid-state NMR spectrum of $\mathrm{V}_{2-}$ propanol is the same as that of other amylose crystalline complexes - e.g., that of $\mathrm{V}_{\text {menthone }}-$, which are isomorphous to $\mathrm{V}_{2 \text {-propanol. }}{ }^{39}$ This observation and that of identical diffraction diagrams for

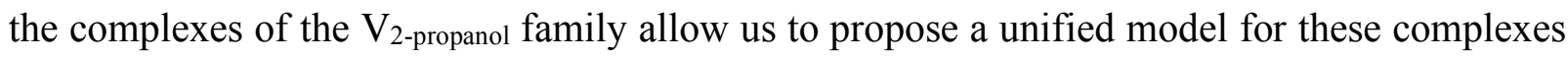
that must have the same rigid skeleton of 7-fold amylose helices while the guest molecules appear as a disorganized background inside the intra- and inter-helical cavities. This scheme explains why crystalline amylose complexed with molecules as different as chloroform, thymol, menthone, 2propanol, etc., still gives the same type of diffraction diagram.

One of the interesting aspects of the $\mathrm{V}_{2 \text {-propanol }}$ single crystals is that they can be converted into the $\mathrm{V}_{\mathrm{H}}$ crystals, that contain $L 6$ single helices, without loosing their perfection and keeping the helical repeat at $0.8 \mathrm{~nm} .{ }^{19,20}$ Based on such observation, our previous hypothesis was that the $\mathrm{V}_{2 \text {-propanol }}$ crystals were also of the 6-fold family. The present work, which rules out this hypothesis, indicates that a smooth transition from 7-fold to lower energy 6-fold helices must occur when 2-propanol is removed from the structure, e.g., by heat annealing. ${ }^{19}$ It is likely that the transition from a 7-fold to a 6 -fold helix is favored by the little conformational difference existing between the glucosyl linkages in the two structures. Indeed, comparisons of the actual geometry of $\alpha$ - and $\beta$-cyclodextrins ${ }^{40}$ and previous calculated 6-fold and 7-fold amylose structures ${ }^{41}$ have confirmed that the conformational angles $\Phi$ and $\Psi$ were nearly the same either in cyclodextrins or in amyloses. For amylose, only as slight modification of the O1-O4 length was calculated to differentiate the glucosyl residues when going from the 7-fold to the 6-fold helix. ${ }^{41}$ Along this line, Sundararajan and Rao, who have studied the conformational transition from $R 7$ to $R 6$ amylose helices, have shown that such interconversion could smoothly occur by slightly modifying the $\tau, \Phi$ and $\Psi$ conformational angles. ${ }^{42}$ In their study, these authors showed that the transformation did not break any intramolecular hydrogen bond and furthermore resulted in a gain in energy. As we know now that the V-amylose crystals do not contain right-handed helices but rather left-handed ones, it remains to be seen whether the calculations of Sundarajan and Rao can be transposed to the conversion of $L 7$ amylose helices into their $L 6$ counterpart.

\section{Perspectives}

In this work, we have refined the skeleton of amylose molecules in the $\mathrm{V}_{2 \text {-propanol }}$ unit cell only using base-plane electron diffraction reflections, which appeared to be enough to 
demonstrate the presence of 7-fold amylose helices and to show their tiling pattern. The validation of the 3-D structure is less certain in the sense that it is only based on modeling and not confirmed by any diffraction data. In order to ascertain the 3-D information of the structure, electron diffraction patterns must be recorded on crystals rotated about selected axes of the reciprocal space, e.g., $a^{*}$ and $b^{*}$.

The molecular description of the guest molecules and their influence on the electron diffraction pattern is another aspect that could be further improved. As it is possible to prepare a variety of $\mathrm{V}$-amylose single crystals that are isomorphous to those of $\mathrm{V}_{2 \text {-propanol, the intensity }}$ variation of their diffraction diagrams should be correlated to the guest density and therefore should help to devise a precise way to subtract the guest contribution from the diffraction datasets. In this respect, the recent preparation of single crystals of amylose complexed with chloroform $\left(d=1.49 \mathrm{~g} / \mathrm{cm}^{3}\right)$, isomorphous to those of 2-propanol $\left(d=0.78 \mathrm{~g} / \mathrm{cm}^{3}\right)$, should yield

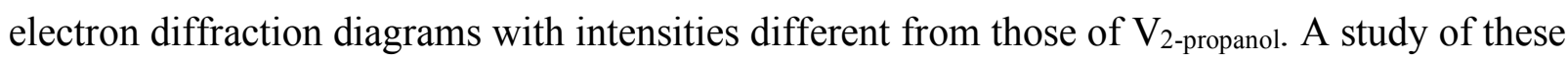
differences should be helpful to improve the structure resolution.

It is interesting to note that amylose single crystals resulting from complexes with $n$-butanol $\left(\mathrm{V}_{n \text {-butanol }}\right)$ display a morphology somewhat similar to that of the crystals of $\mathrm{V}_{2 \text {-propanol. }}$ Nevertheless, their electron diffraction diagrams, which are also very rich, are different. Whereas all authors refer to these crystals as resulting from the packing of 6-fold helices rather than 7fold, ${ }^{43,44}$ no crystal structure has been proposed yet. The protocol reported in this paper is presently being applied to the resolution of the $\mathrm{V}_{n \text {-butanol }}$ crystal structure. The same approach is also followed to confirm the structure of $\mathrm{V}_{\alpha \text {-naphthol }}$ crystals which were shown to consist of 8fold amylose helices. ${ }^{45}$

Acknowledgment. We thank K. Gessler for the gift of a sample of synthetic amylose. M. B. C.'s contribution to this work was a part of his $\mathrm{PhD}$ study under the joint supervision by J-L. P. and N. Silveira (LINDM, Porto Alegre, Brazil). M.B.C. received a fellowship from the Coordenação de Aperfeiçoamento de Pessoal de Nivel Superior (CAPES) from Brazil. The help of A. D. French for valuable discussions during the writing of this report is also acknowledged. 


\section{References}

(1) Tomasik, P.; Schilling, C. H. Adv. Carbohydr. Chem. Biochem. 1988, 53, 263-343.

(2) Kuge, T.; Takeo, K. Agr. Biol. Chem. 1968, 32, 1232-1238.

(3) Tomasik, P.; Schilling, C. H. Adv. Carbohydr. Chem. Biochem. 1988, 53, 345-426.

(4) Putseys, J. A.; Lamberts, L.; Delcour, J. A. J. Cereal Sci. 2010, 51, 238-247.

(5) Buléon, A.; Véronèse, G.; Putaux, J-L. Aust. J. Chem. 2007, 60, 706-718.

(6) Jenkins, P. J.; Donald, A. M. Int. J. Biol. Macromol. 1995, 17, 315-321.

(7) Buléon, A.; Duprat, F.; Booy F. P.; Chanzy, H. Carbohydr. Polym. 1984, 4, 161-173.

(8) Imberty, A.; Chanzy, H.; Pérez, S.; Buléon, A.; Tran, V. J. Mol. Biol. 1988, 201, 365-378.

(9) Popov, D.; Buléon, A.; Burghammer, M.; Chanzy; H.; Montesanti, N.; Putaux, J-L.; Potocki-Véronèse, G.; Riekel, C. Macromolecules 2009, 42, 1167-1174.

(10) Katz, J. R.; van Itallie, T. B. Z. Physikal. Chem. 1930, 150(Abt. A), 90-99.

(11) Senti, F. R. High-amylose corn starch: its production, properties, and uses. In Starch: Chemistry and Technology, Whistler, R. L., Paschall, E. F. Bemiller, J. N., Roberts, H. J. Eds. Academic Press: New-York, 1967, Vol. II, p. 511.

(12) Zaslow, B.; Murphy, V. G.; French, A. D. Biopolymers, 1974, 13, 779-790.

(13) Rappenecker, G.; Zugenmaier, P. Carbohydr. Res. 1981, 89, 11-19.

(14) Brisson, J.; Chanzy, H.; Winter, W. T. Int. J. Biol. Macromol. 1991, 13, 31-39.

(15) Bluhm, T. L.; Zugenmaier, P. Carbohydr. Res. 1981, 89, 1-10.

(16) Winter, W. T.; Sarko, A. Biopolymers 1974, 13, 1461-1482.

(17) Yamashita, Y.; Ryugo, J; Monobe, K. J. Electron Microsc. 1973, 22, 19-26.

(18) Yamashita, Y.; Hirai, N. J. Polym. Sci. Part A2, 1966, 4, 161-171.

(19) Buléon, A.; Delage, M. M.; Brisson, J.; Chanzy, H. Int. J. Biol. Macromol. 1990, 12, 25-33.

(20) Putaux, J-L.; Cardoso, M. B.; Dupeyre, D.; Morin, M.; Nulac, A. Hu, Y. Macromol. Symp. 2008, 273, 1-8.

(21) Helbert, W. Doctoral Dissertation, Joseph Fourier University of Grenoble, France, 1994.

(22) Nuessli, J.; Putaux, J-L.; Le Bail; P.; Buléon, A. Int. J. Biol. Macromol. 2003, 33, 227-234.

(23) Heinemann, C.; Zinsli, M.; Renggli, A.; Escher, F.; Conde-Petit, B. LWT 2005, 38, 885-894.

(24) Zaslow, B. Biopolymers 1963, 1, 165-169.

(25) Takeo, K.; Kuge, T. Agr. Biol. Chem. 1969, 33, 1174-1180.

(26) Simpson, T. D.; Dintzis, F. R.; Taylor, N. W. Biopolymers 1972, 11, 2591-2600.

(27) Clark, M.; Cramer III, R. D.; Van Opdenbosch, N. J. Comp. Chem. 1989, 10, 982-1012.

(28) French, A. D.; Murphy, V. G. Cereal Food World 1977, 22, 61-70.

(29) Zugenmaier, P.; Sarko, A. Biopolymers, 1973, 12, 435-444.

(30) Rappé, A. K.; Casewit, C. J., Colwell, K. S.; Goddard, W. A.; Skiff, W. M. J. Am. Chem. Soc. 1992, 114, 10024-10035.

(31) Cerius ${ }^{2}$ program from Accelrys Inc, San Diego, Ca.

(32) Dauchez, M.; Derreumaux, P.; Vergoten, G. J. Comp. Chem. 1992, 14, 263-277.

(33) Fraser, R. D. B.; MacRae, T. P.; Suzuki, E. J. Appl. Cryst. 1978, 11, 693-694. 
(34) Sheldrick, G. M. SHELX-97, a program for the refinement of single-crystals diffraction data, University of Göttingen, Göttingen, Germany, 1997.

(35) The parameters for the elastic atomic scattering factors of electrons for $\mathrm{H}, \mathrm{C}$ and $\mathrm{O}$ atoms are given in Supplementary Information Table S1. This list, adapted for SHELX-97, is derived from Table 4.3.2.2 in the International Tables of Crystallography, Volume C, Kluwer Academic Publishers, London 2004, p 282.

(36) Connolly, M. L. J. Am. Chem. Soc. 1985, 107, 1118-1124.

(37) Cremer, D.; Pople, J. A. J. Am. Chem. Soc. 1975, 97, 1354-1358.

(38) Bear, R. S. J. Am. Chem. Soc. 1944, 66, 2122-2123.

(39) Le Bail, P.; Rondeau, C.; Buléon, A. Int. J. Biol. Macromol. 2005, 35, 1-7.

(40) French, A. D.; Johnson, G. P. Carbohydr. Res. 2007, 342, 1223-1237.

(41) French, A D.: Murphy; V. G. Polymer, 1977, 18, 489-494.

(42) Sundararajan, P. R.; Rao, V. S. R. Biopolymers 1969, 8, 313-323.

(43) Rundle, R. E.; Edwards, F. C. J. Am. Chem. Soc. 1943, 65, 2200-2203.

(44) Yamashita, Y. J. Polym. Sci. Part A 1965, 3, 3251-3260.

(45) Cardoso, M. B.; Putaux J-L.; Nishiyama, Y.; Helbert, W.; Hÿtch, M., Silveira, N. P.; Chanzy, H. Biomacromolecules 2007, 8, 1319-1326. 


\section{Tables and Figures}

Table 1. Selection of the best models for each rigid helical amylose system as a function of the minimum packing energy and best reliability factor $R_{2}$.

\begin{tabular}{ccc}
\hline Helix $^{\mathrm{a}}$ & Packing energy $^{\mathrm{b}}$ & $R 2^{\mathrm{c}}$ \\
\hline L6gg & -25.0 & 0.597 \\
L6gt & -24.5 & 0.632 \\
$L 6 \operatorname{tg}$ & -23.6 & 0.653 \\
$R 6 g g$ & -27.1 & 0.778 \\
$R 6 g t$ & -26.0 & 0.835 \\
$R 6 \operatorname{tg}$ & -26.6 & 0.758 \\
$L 7 g g$ & -49.1 & 0.214 \\
$L 7 g t$ & -48.6 & 0.212 \\
$L 7 t g$ & -48.4 & 0.354 \\
$R 7 g g$ & -41.6 & 0.317 \\
$R 7 g t$ & -41.2 & 0.546 \\
$R 7 \operatorname{tg}$ & -42.2 & 0.307 \\
$L 8 g g$ & $>1000$ & 0.546 \\
$L 8 g t$ & 529 & 0.412 \\
$L 8 \operatorname{tg}$ & 634 & 0.604 \\
$R 8 g g$ & $>1000$ & 0.652 \\
$R 8 g t$ & $>1000$ & 0.656 \\
$R 8 \operatorname{tg}$ & $>1000$ & 0.644 \\
\hline & & \\
\hline & &
\end{tabular}

${ }^{a}$ Left- and right-handed ( $L$ and $R$, respectively) 6-, 7- and 8-fold helices with the hydroxymethyl groups in the $g g, g t$ and $t g$ positions.

${ }^{\mathrm{b}}$ Packing energy in $\mathrm{kJ} /$ glucosyl unit.

${ }^{\mathrm{c}}$ see the text for the definition of $R_{2}$. 
Table 2. $R 1$-factors of the $R 7 g g, R 7 \operatorname{tg} L 7 g t$ and $L 7 g t$, models after relaxation and refinement using SHELX.

\begin{tabular}{cccc}
\hline Helix & Guest solvent & $\begin{array}{c}71 \text { reflections } \\
(\mathrm{d}>0.3 \mathrm{~nm})\end{array}$ & $\begin{array}{c}108 \text { reflections } \\
(\mathrm{d}>0.2 \mathrm{~nm})\end{array}$ \\
\hline$R 7 g g$ & continuous & 0.46 & n. c. \\
$R 7 \mathrm{tg}$ & continuous & 0.44 & n. c. \\
$L 7 g t$ & continuous & 0.45 & n. c. \\
& continuous & 0.28 & 0.36 \\
$L 7 g g$ & continuous + BASF procedure & 0.23 & 0.26 \\
& explicit molecules & 0.10 & 0.18 \\
\hline
\end{tabular}

${ }^{\mathrm{a}}$ see text for the description of the intensity scaling procedure.

n. c.: not calculated 
Table 3. Selected geometrical parameters of the 7 glucosyl residues constituting the 7-fold left-handed amylose helix.

\begin{tabular}{ccccccccccccc}
\hline $\begin{array}{c}\text { Residue } \\
\text { number }\end{array}$ & \multicolumn{2}{c}{$\Phi\left(^{\circ}\right)$} & \multicolumn{2}{c}{$\Psi\left(^{\circ}\right)$} & \multicolumn{2}{c}{$\tau\left(^{\circ}\right)$} & \multicolumn{2}{c}{$\omega\left(^{\circ}\right)$} & & $\theta\left(^{\circ}\right)$ & \multicolumn{2}{c}{ O1-O4 (nm) } \\
& & $\mathrm{a}$ & $\mathrm{b}$ & $\mathrm{a}$ & $\mathrm{b}$ & $\mathrm{a}$ & $\mathrm{b}$ & $\mathrm{a}$ & $\mathrm{b}$ & $\mathrm{a}$ & $\mathrm{b}$ & $\mathrm{b}$ \\
\hline 1 & $113(4)$ & $113(2)$ & $-142(3)$ & $-131(3)$ & $114(2)$ & $112(2)$ & $8(5)$ & $-82(3)$ & $2(3)$ & $0.0(19)$ & 0.45 \\
2 & $104(3)$ & $96(3)$ & $-134(3)$ & $-136(3)$ & $117(2)$ & $114(1)$ & $-11(4)$ & $-71(3)$ & $0(3)$ & $14(3)$ & 0.42 \\
3 & $113(4)$ & $129(2)$ & $-116(3)$ & $-126(2)$ & $120(2)$ & $112(2)$ & $-49(4)$ & $-61(3)$ & $18(3)$ & $12(3)$ & 0.44 \\
4 & $105(3)$ & $125(3)$ & $-139(3)$ & $-122(3)$ & $115(2)$ & $113(2)$ & $-82(4)$ & $11(3)$ & $0(3)$ & $12(2)$ & 0.43 \\
5 & $103(4)$ & $82(3)$ & $-124(4)$ & $-94(3)$ & $119(2)$ & $115(2)$ & $55(4)$ & $-30(3)$ & $3(3)$ & $14(2)$ & 0.44 \\
6 & $115(3)$ & $120(2)$ & $-155(3)$ & $-160(3)$ & $112(2)$ & $112(1)$ & $-136(3)$ & $-99(3)$ & $6(3)$ & $7(2)$ & 0.48 \\
7 & $124(3)$ & $100(3)$ & $-114(3)$ & $-108(3)$ & $119(2)$ & $112(2)$ & $-73(3)$ & $-97(3)$ & $13(3)$ & $17(3)$ & 0.39 \\
\hline
\end{tabular}

For the angles $\Phi, \Psi, \tau$ and $\omega$, column 'a' corresponds to a SHELX refinement with the solvent taken as a continuum (model 2) and column 'b' with explicit solvent molecules (model 3). The distance O1-O4 corresponds to model 3. In the case of the initial L7gg model, the rigid helices were built with $\Phi=99.5^{\circ}, \Psi=-125.8^{\circ}, \theta=3.7^{\circ}$ and $\omega=-60^{\circ}$.

The glucosidic torsion angles $\Phi$ and $\Psi$, which describe the relative orientation of adjacent glucosyl residues in a given amylose chain, are defined by (O5-C1-O1-C4) and (C1-O1-C4-C5), respectively, and the bond angle $\tau$ is defined as (C1-O1-C4). The hydroxymethyl conformation $\omega$ is defined as the torsion angle (O5-C5-C6-O6) with $\omega_{\mathrm{gg}}=-60^{\circ}, \omega_{\mathrm{gt}}=+60^{\circ}$ and $\omega_{\mathrm{tg}}=180^{\circ}$. The puckering parameter $\theta$ is defined as in ref. 37 . 


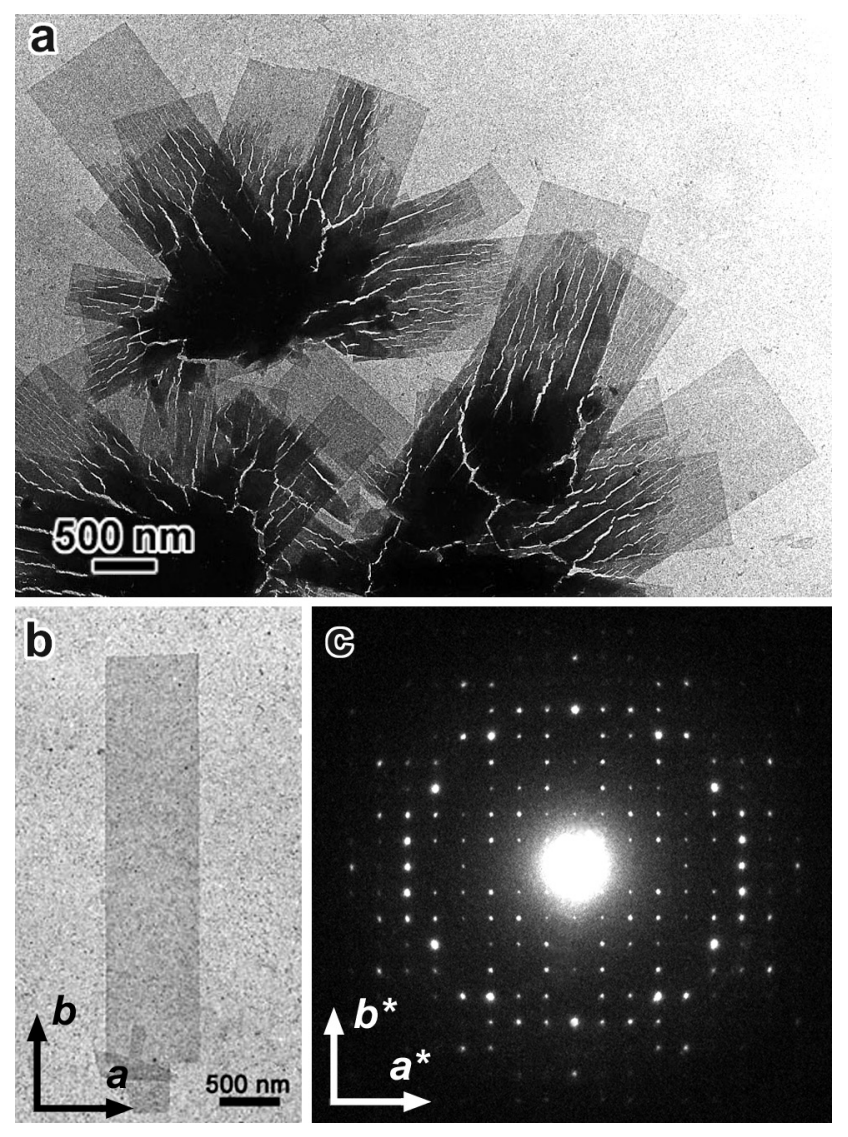

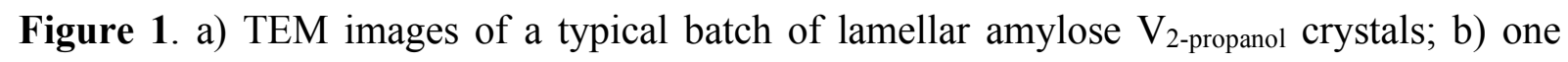
crystalline lamella; c) electron diffraction diagram recorded on one square micron of the sample under frozen-hydrated conditions, properly oriented with respect to the crystal. 


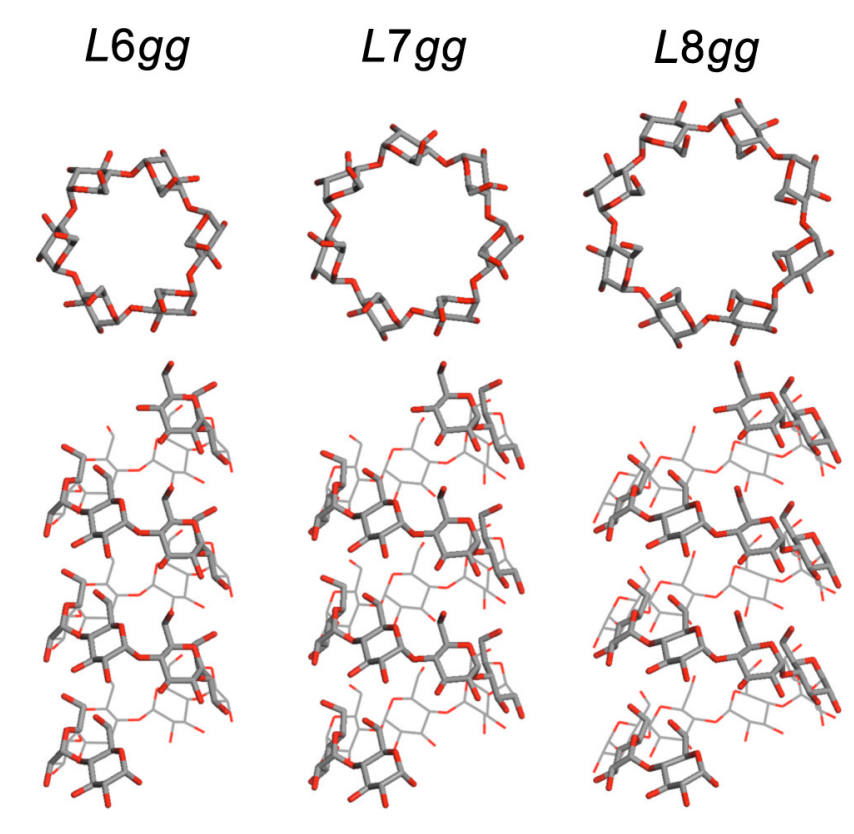

Figure 2. Examples of 6-, 7- and 8-fold left-handed amylose helices with hydroxymethyl groups in the gg conformation, projected parallel and perpendicular to the helix axis. 

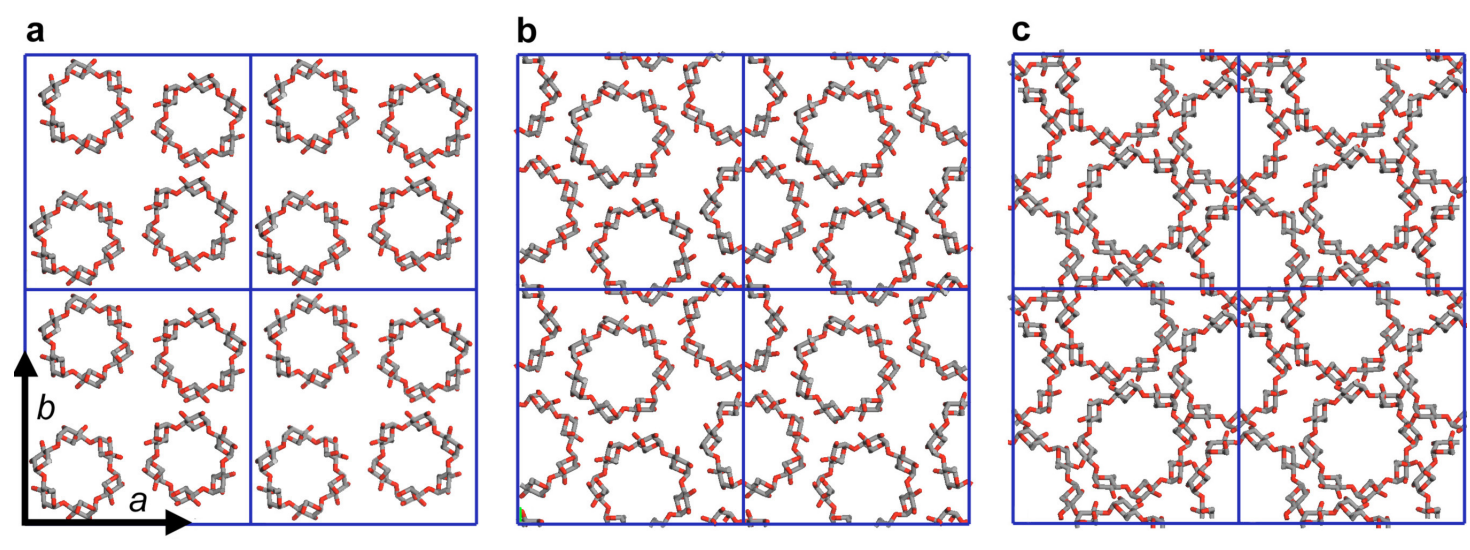

Figure 3. Models of packing of 6-, 7-, and 8-fold left-handed helices with hydroxymethyl groups in the $g g$ conformation, projected on the $(a, b)$ plane of the unit cell. 

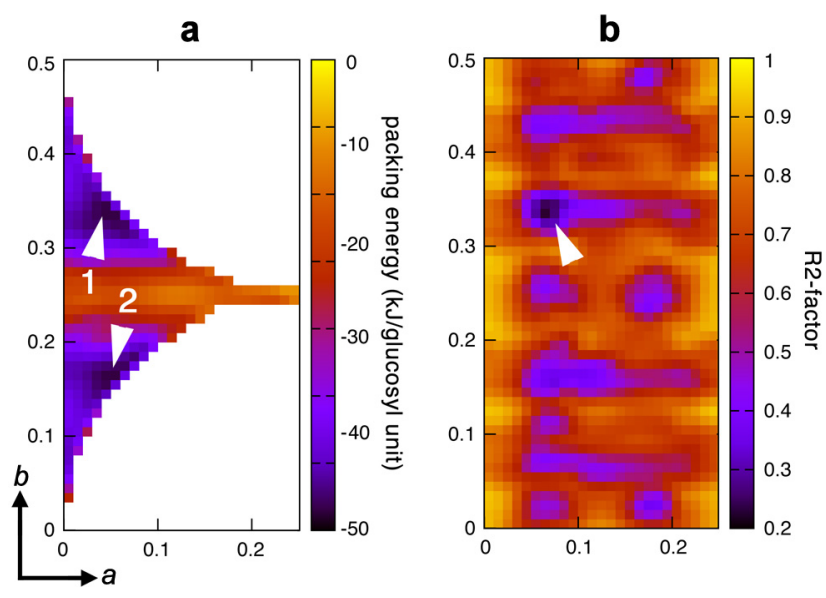

Figure 4. a) Color map of the packing energy of 7-fold left-handed helices with hydroxymethyl groups in the $g g$ conformation $(L 7 g g)$ when they are translated and rotated within $1 / 8^{\text {th }}$ of the $(a, b)$ plane of the unit cell. Arrows 1 and 2 correspond to energy minima. b) Color map of the $R_{2}$-factor between the observed and calculated electron diffraction diagram resulting from the translation and rotation of $L 7 g g$ helices within $1 / 8^{\text {th }}$ of the $(a, b)$ plane of the unit cell. The helix position in the unit cell is indicated with fractional coordinates. 


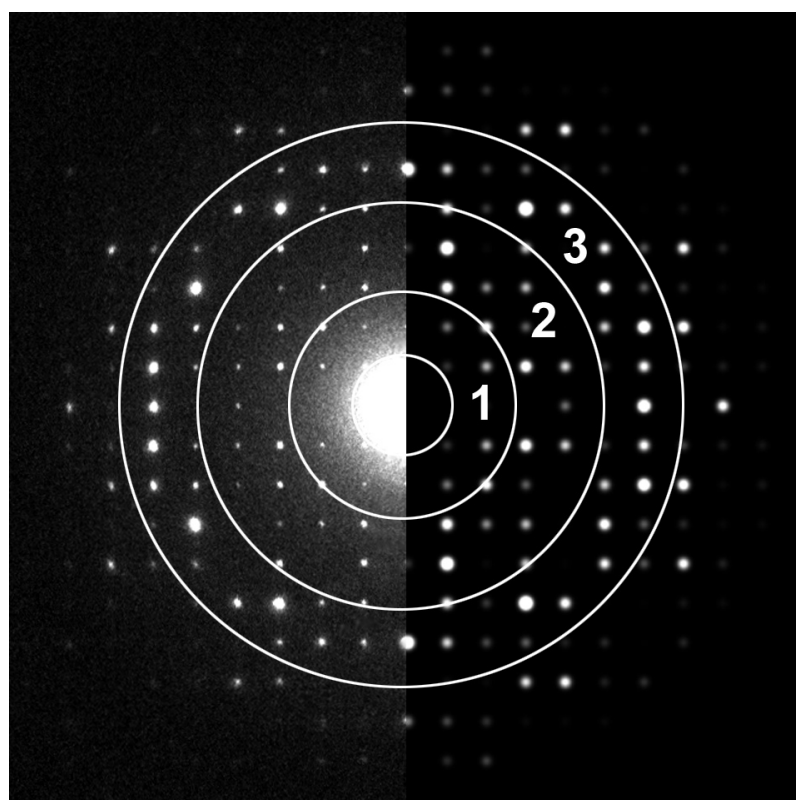

Figure 5. Observed (left) and calculated (right) electron diffraction diagrams of the selected L7gg model after initial SHELX-97 refinement, taking the solvent as a continuum of density 0.8. In zone 2, the calculated intensities are systematically larger than the observed ones. In zones 1 and 3, there is a better agreement between the observed and calculated intensities. 

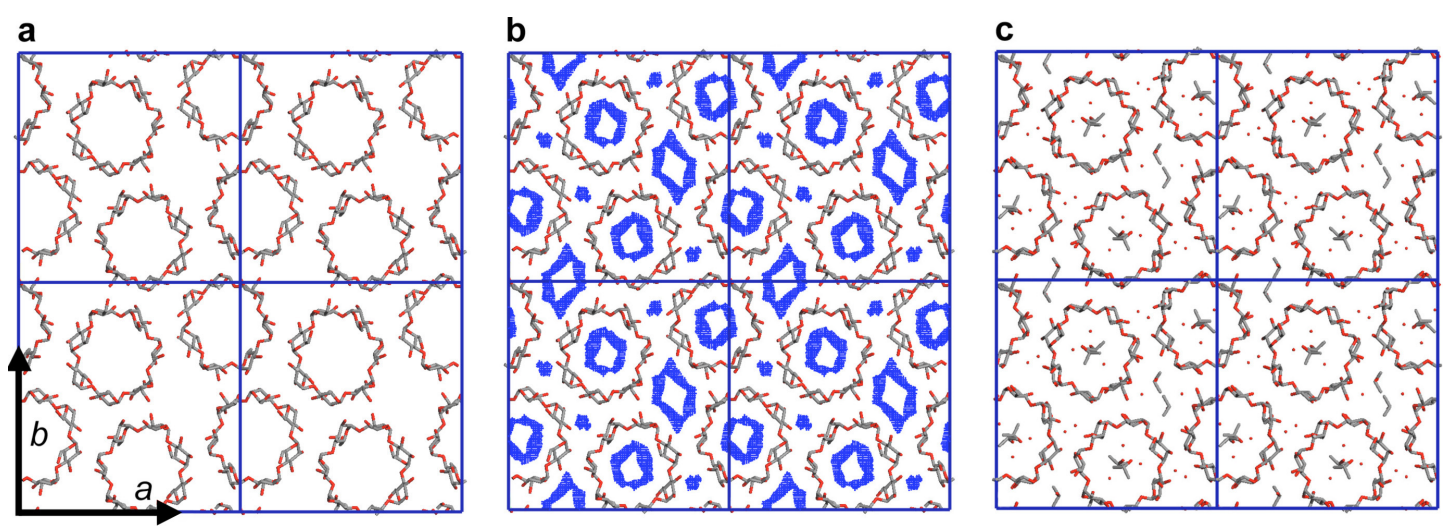

Figure 6. Projections on the ( $(a, b)$ plane of the $L 7 g g$ structure: a) packing of the helices resulting from the refinement with adjustable scale factors; b) as in a, but with Connolly surfaces showing the voids accessible to the ligands; c) final refinement, after incorporation of explicit 2-propanol and water molecules located inside and between the helices. 

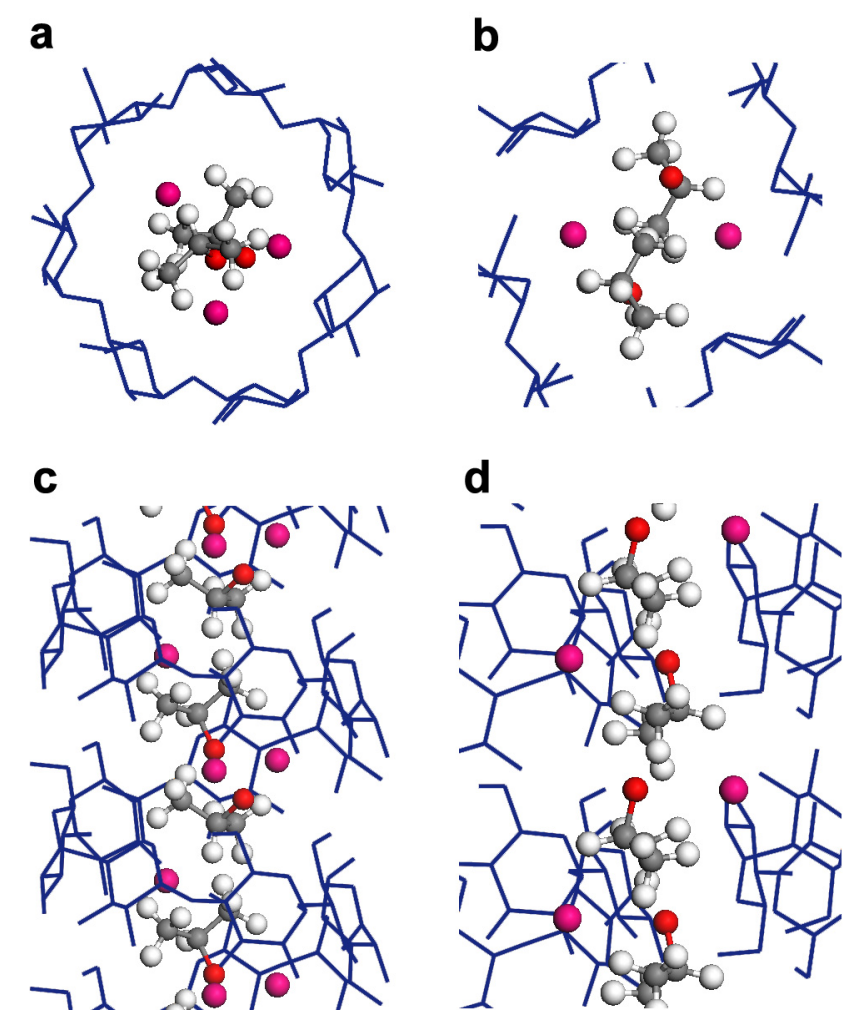

Figure 7. Axial ( $a$ and b) and longitudinal ( $c$ and d) views of the helices in the final structure showing a possible position of the guest 2-propanol and water molecules inside (a and c) and between ( $b$ and $d)$ the helical cavity. 


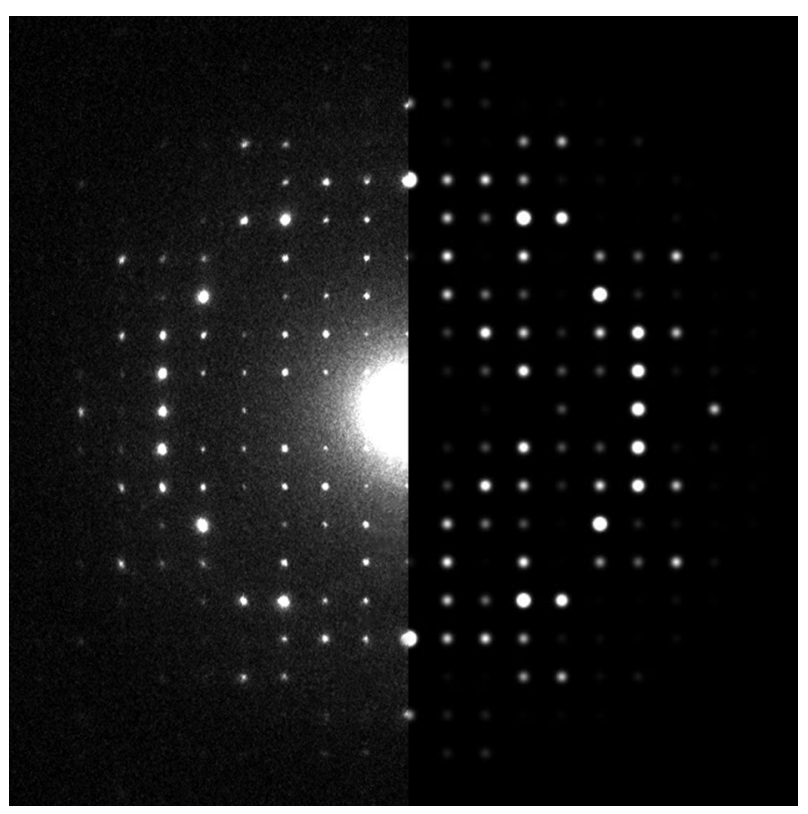

Figure 8. Composite electron diffraction diagrams showing the observed (left) and calculated (right) electron diffraction diagrams of the final structure of $\mathrm{V}_{2 \text {-propanol }}$ crystal. 


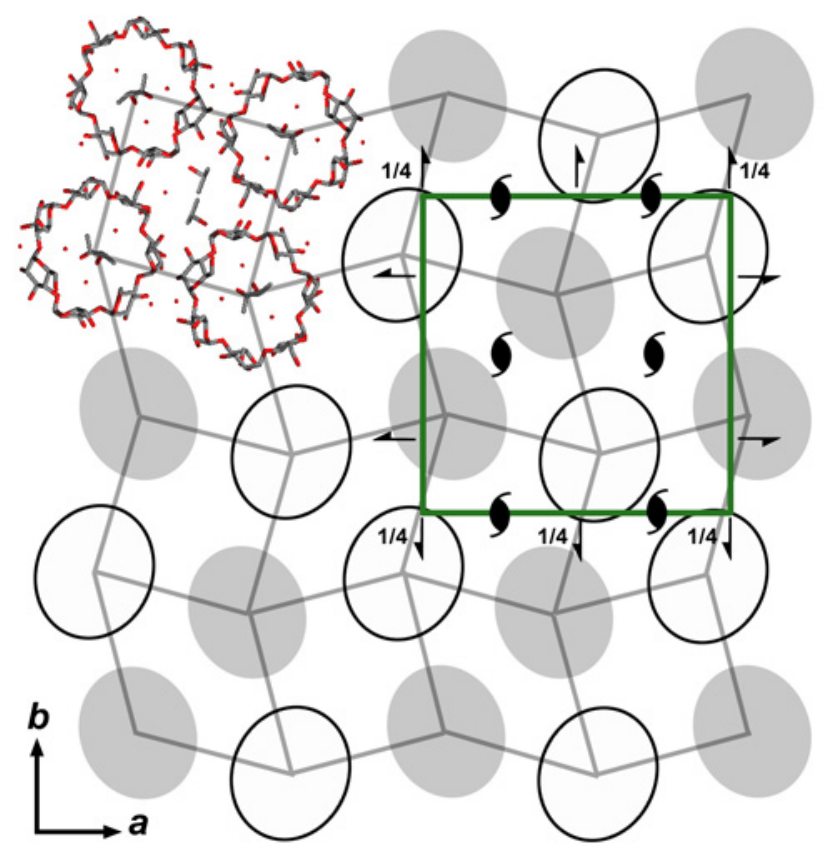

Figure 9. Tiled arrangement of the amylose helices, with their complexing solvent in the $(a, b)$ plane of the unit cell. The grey and white ellipses correspond to "up" and "down" helices, respectively, as shown in the upper left of the figure. The symmetry axes are represented in the unit cell outlined in green. The grid describes the alternating motifs of 4 amylose helices in close-packed hexagonal arrangement together with 4 helices in a nearly square arrangement. 


\section{Supporting Information}

\section{Correction of the scattered intensities by taking atomic scattering factors into account}

The expression of the atomic scattering factors of electrons for neutral atoms is:

$$
f(s)=\sum_{i} a_{i} \exp \left(-b_{i} s^{2}\right)
$$

where $s$ is the scattering vector $\sin \theta / \lambda, \theta$ being half the scattering angle and $\lambda$ the electron wavelength. The values of the $\left(a_{i}, b_{i}\right)$ coefficients are given in Table 4.3.2.2 of International Tables for Crystallography. ${ }^{37}$ The scattering factors of carbon, oxygen and hydrogen atoms found in this table are shown in Figure S1a. The solvent (i.e., 2-propanol) was assumed to have a constant scattering power corresponding to the sum of the scattering factors at $s=0$ with a composition $\mathrm{H}: \mathrm{C}: \mathrm{O}$ of $8: 3: 1$ and a density of $0.8 \mathrm{~g} / \mathrm{cm}^{3}$. The mean scattering density of the solvent, $\rho_{\mathrm{c}}$, is given by:

$$
\rho_{e}=\frac{N_{A} \rho}{M_{w} 10^{24}} \sum_{j} n_{j} f_{j}(0)
$$

where $N_{A}$ is Avogadro's number, $\rho$ and $M_{w}$ are the density and the molecular weight of the solvent, respectively, and $n_{j}$ is the number of atoms of the $j^{\text {th }}$ atom in the formula. $f_{j}(0)$ is the atomic scattering factor at angle 0 of the $j^{\text {in }}$ atom.

According to Fraser et al., ${ }^{35}$ the expression of the modified scattering factor $f^{\prime}(s)$ is:

$$
f^{\prime}(s)=f(s)-v \rho_{e} \exp \left(-4 \pi v^{\frac{2}{3}} s^{2}\right)
$$

Assuming a density of $0.8 \mathrm{~g} / \mathrm{cm}^{3}$ and a molecular formula of $\mathrm{H} 8 \mathrm{C} 3 \mathrm{O} 1$, the atomic scattering factor is given in Figure S1b. Each scattering curve was fitted with 4 Gaussian functions whose parameters were then used in the "SFAC" procedure of SHELX. The values of the resulting $\left(a_{i}, b_{i}\right)$ coefficients are listed in Table $\mathbf{S 1 .}$ 

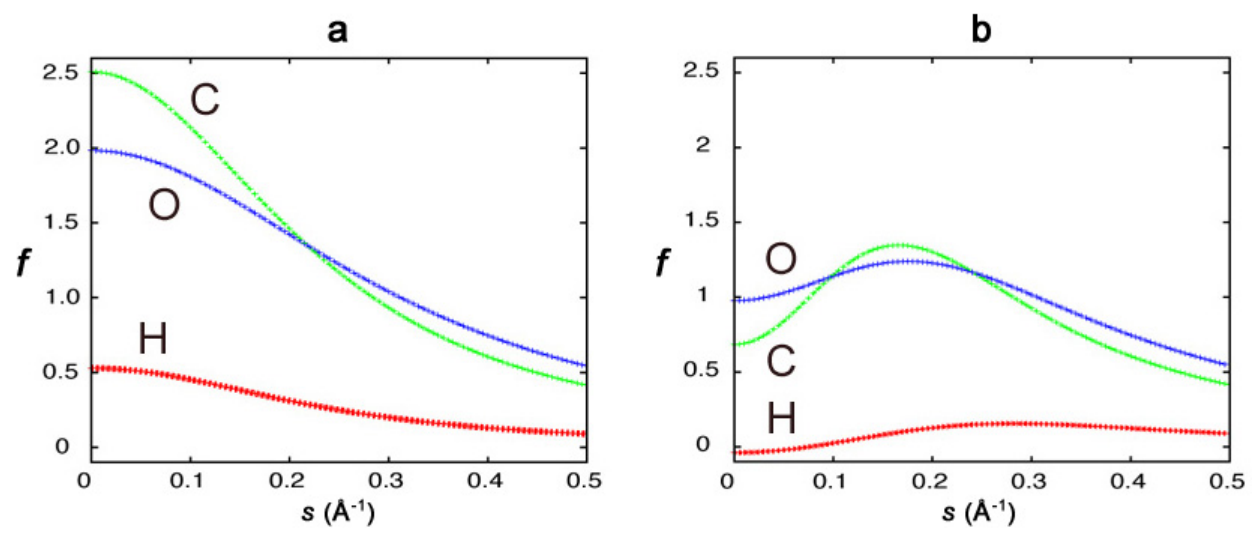

Figure S1. Scattering factors for carbon, oxygen and hydrogen atoms: a) without guest solvent; b) with guest solvent taken as a continuous medium.

Table S1. Parameters for the Gaussian analytical form of the atomic scattering factors.

\begin{tabular}{ccccccccc}
\hline atom & $a_{0}$ & $b_{0}$ & $a_{1}$ & $b_{1}$ & $a_{2}$ & $b_{2}$ & $a_{3}$ & $b_{3}$ \\
\hline $\mathrm{H}^{\mathrm{a}}$ & 0.1059 & 1.7392 & 0.1382 & 8.2978 & 0.1644 & 15.8646 & 0.1204 & 38.5920 \\
$\mathrm{H}^{\mathrm{b}}$ & 1.4873. & 6.1705 & -2.7798 & 8.6907 & 3.8585 & 13.9664 & -2.6053 & 17.7810 \\
$\mathrm{C}^{\mathrm{a}}$ & 0.2934 & 0.9615 & 0.7985 & 6.1270 & 1.0588 & 18.5408 & 0.3581 & 50.2303 \\
$\mathrm{C}^{\mathrm{b}}$ & 0.6061 & 2.1709 & 1.0008 & 11.2561 & 0.6370 & 24.6959 & -1.5603 & 62.3221 \\
$\mathrm{O}^{\mathrm{a}}$ & 0.3168 & 0.7535 & 0.7494 & 4.4016 & 0.7126 & 12.6265 & 0.2046 & 32.4451 \\
$\mathrm{O}^{\mathrm{b}}$ & 0.6699 & 1.7055 & 0.9168 & 8.7363 & 0.2878 & 20.8208 & -0.8981 & 41.6749 \\
\hline
\end{tabular}

a corresponding to Figure S1a.

${ }^{\circ}$ corresponding to Figure S1b. 
Table S2. List of observed reflections and their intensities $\left(I_{\mathrm{obk}}\right)$ in the electron diffraction patterns of $\mathrm{V}_{\text {2propana }}$ crystals, down to a resolution of $0.214 \mathrm{~nm}$. The first 71 reflections to a resolution of $0.303 \mathrm{~nm}$ (reflection 850) were used for the initial refinement.

\begin{tabular}{|c|c|c|}
\hline$h k l$ & $I_{\mathrm{obs}}$ & $\sigma$ \\
\hline 100 & 2.95 & 1.54 \\
\hline 020 & 0.00 & 1.00 \\
\hline 200 & 9.96 & 1.70 \\
\hline 120 & 8.19 & 2.42 \\
\hline 210 & 8.90 & 1.10 \\
\hline 220 & 58.52 & 2.34 \\
\hline 130 & 56.19 & 1.67 \\
\hline 310 & 34.66 & 1.84 \\
\hline 230 & 40.25 & 2.28 \\
\hline 320 & 21.28 & 0.52 \\
\hline 040 & 15.39 & 1.00 \\
\hline 140 & 11.53 & 1.32 \\
\hline 400 & 5.88 & 0.56 \\
\hline 410 & 42.74 & 3.58 \\
\hline 330 & 13.86 & 0.85 \\
\hline 240 & 6.74 & 1.34 \\
\hline 420 & 0.71 & 0.36 \\
\hline 340 & 0.97 & 0.28 \\
\hline 150 & 12.28 & 1.01 \\
\hline 430 & 56.31 & 3.08 \\
\hline 510 & 36.80 & 2.03 \\
\hline 250 & 47.68 & 0.37 \\
\hline 520 & 20.12 & 1.59 \\
\hline 440 & 0.61 & 0.31 \\
\hline 350 & 73.95 & 7.42 \\
\hline 530 & 90.82 & 5.81 \\
\hline 060 & 171.24 & 5.00 \\
\hline 160 & 214.86 & 13.21 \\
\hline 600 & 360.11 & 25.80 \\
\hline 610 & 38.8 & 1.26 \\
\hline 260 & 97.49 & 4.20 \\
\hline 450 & 27.23 & 2.56 \\
\hline 620 & 53.47 & 4.18 \\
\hline 540 & 81.73 & 6.4 \\
\hline 360 & 7.81 & 1.04 \\
\hline 630 & 30.2 & 2.05 \\
\hline 170 & 3.15 & 0.97 \\
\hline 550 & 3.00 & 1.64 \\
\hline 460 & 17.35 & 1.40 \\
\hline 270 & 34.19 & 1.80 \\
\hline 710 & 2.67 & 0.93 \\
\hline
\end{tabular}




\begin{tabular}{|c|c|c|}
\hline 640 & 1.61 & 1.39 \\
\hline 720 & 0.82 & 0.48 \\
\hline 370 & 3.25 & 0.66 \\
\hline 730 & 25.20 & 2.82 \\
\hline 560 & 0.28 & 0.14 \\
\hline 650 & 2.61 & 0.87 \\
\hline 080 & 36.72 & 1.00 \\
\hline 180 & 3.42 & 0.34 \\
\hline 470 & 45.92 & 0.49 \\
\hline 280 & 1.98 & 0.49 \\
\hline 740 & 39.60 & 4.28 \\
\hline 800 & 35.59 & 5.67 \\
\hline 810 & 5.73 & 0.75 \\
\hline 820 & 4.91 & 0.57 \\
\hline 380 & 0.89 & 0.28 \\
\hline 660 & 0.44 & 0.22 \\
\hline 570 & 2.84 & 0.51 \\
\hline 750 & 2.34 & 1.03 \\
\hline 830 & 1.30 & 0.80 \\
\hline 480 & 3.25 & 0.41 \\
\hline 190 & 0.57 & 0.50 \\
\hline 840 & 1.46 & 0.52 \\
\hline 290 & 1.05 & 0.53 \\
\hline 670 & 2.08 & 0.40 \\
\hline 910 & 4.90 & 0.32 \\
\hline 760 & 4.22 & 0.85 \\
\hline 390 & 1.08 & 0.40 \\
\hline 580 & 0.64 & 0.32 \\
\hline 920 & 7.13 & 0.65 \\
\hline 850 & 0.48 & 0.42 \\
\hline 930 & 0.78 & 0.42 \\
\hline 490 & 1.23 & 0.47 \\
\hline 0100 & 0.71 & 1.00 \\
\hline 1100 & 3.21 & 0.28 \\
\hline 770 & 0.00 & 0.00 \\
\hline 680 & 9.28 & 0.95 \\
\hline 940 & 4.47 & 0.44 \\
\hline 2100 & 2.33 & 0.54 \\
\hline 860 & 0.40 & 0.34 \\
\hline 1000 & 0.00 & 0.00 \\
\hline 590 & 0.30 & 0.15 \\
\hline 1010 & 2.08 & 0.72 \\
\hline 3100 & 1.30 & 0.39 \\
\hline 1020 & 0.59 & 0.30 \\
\hline 950 & 2.79 & 0.46 \\
\hline 1030 & 0.34 & 0.18 \\
\hline 780 & 0.59 & 0.19 \\
\hline 4100 & 0.57 & 0.30 \\
\hline 870 & 0.00 & 0.00 \\
\hline 690 & 0.71 & 0.42 \\
\hline
\end{tabular}




$\begin{array}{ccc}960 & 0.39 & 0.13 \\ 1040 & 0.38 & 0.23 \\ 5100 & 1.52 & 0.92 \\ 1050 & 0.42 & 0.21 \\ 880 & 2.33 & 1.19 \\ 790 & 1.53 & 0.44 \\ 970 & 0.19 & 0.16 \\ 6100 & 3.29 & 0.27 \\ 1060 & 0.00 & 0.00 \\ 890 & 0.30 & 0.26 \\ 980 & 1.80 & 0.55 \\ 7100 & 0.62 & 0.31 \\ 1070 & 5.06 & 0.70 \\ 990 & 1.16 & 0.59 \\ 8100 & 0.82 & 0.27 \\ 1080 & 0.59 & 0.30 \\ 9100 & 0.14 & 0.12\end{array}$

